Proceedings of the Edinburgh Mathematical Society (2003) 46, 415-420 (C)

DOI:10.1017/S0013091502000524 Printed in the United Kingdom

\title{
NUMERICAL INDEX AND THE DAUGAVET PROPERTY FOR $L_{\infty}(\mu, X)$
}

\author{
MIGUEL MARTÍN AND ARMANDO R. VILLENA \\ Departamento de Análisis Matemático, Facultad de Ciencias, \\ Universidad de Granada, 18071-Granada, Spain \\ (mmartins@ugr.es; avillena@ugr.es)
}

(Received 27 May 2002)

\begin{abstract}
We prove that the space $L_{\infty}(\mu, X)$ has the same numerical index as the Banach space $X$ for every $\sigma$-finite measure $\mu$. We also show that $L_{\infty}(\mu, X)$ has the Daugavet property if and only if $X$ has or $\mu$ is atomless.
\end{abstract}

Keywords: Daugavet property; numerical index; numerical radius

2000 Mathematics subject classification: Primary 46B20; 47A12

\section{Introduction}

The concept of numerical index was first suggested by Lumer in 1968. Since then a lot of attention has been paid to this quantitative characteristic of a Banach space. Classical references here are $[\mathbf{2}, \mathbf{3}]$. For recent results we refer the reader to $[\mathbf{7}-\mathbf{9}]$.

Here and subsequently, for a real or complex Banach space $X$, we write $B_{X}$ for the closed unit ball and $S_{X}$ for the unit sphere of $X$. The dual space is denoted by $X^{*}$ and the Banach algebra of all continuous linear operators on $X$ is denoted by $L(X)$. The numerical range of $T \in L(X)$ is

$$
V(T)=\left\{x^{*}(T x): x \in S_{X}, x^{*} \in S_{X^{*}}, x^{*}(x)=1\right\} .
$$

The numerical radius is the seminorm defined on $L(X)$ by

$$
v(T)=\sup \{|\lambda|: \lambda \in V(T)\}
$$

for each $T \in L(X)$. The numerical index of the space $X$ is defined by

$$
n(X)=\inf \left\{v(T): T \in S_{L(X)}\right\} .
$$

In this paper we prove that the numerical index of $L_{\infty}(\mu, X)$ coincides with the numerical index of $X$ whenever $\mu$ is a $\sigma$-finite measure and $X$ is an arbitrary Banach space. It should be pointed out that this result is analogous to those given in [9] for $C(K, X)$, $L_{1}(\mu, X)$ and $l_{\infty}(X)$. 
The numerical index is related to the so-called Daugavet property (see $[\mathbf{9}]$ ). The remarkable fact that every compact operator $T$ on $C[0,1]$ satisfies

$$
\|\operatorname{Id}+T\|=1+\|T\|,
$$

where Id stands for the identity, goes back to Daugavet [4] and this equality has currently become known as the Daugavet equation. We follow $[6]$ in saying that a Banach space $X$ has the Daugavet property if every rank-one operator $T \in L(X)$ satisfies (DE). In such a case, it is known that every weakly compact operator on $X$ also satisfies the Daugavet equation. Consequently, this definition is equivalent to that given in [1] . For recent results we refer the reader to $[\mathbf{6}, \mathbf{1 1}, \mathbf{1 2}]$ and the references therein.

It is known that $C(K)$ has the Daugavet property for every perfect compact space $K$, and $L_{1}(\mu), L_{\infty}(\mu)$ have the Daugavet property for every atomless positive measure $\mu$ (see $[\mathbf{1 2}]$ for a detailed account of these facts). The non-commutative versions have recently been obtained in [10]. It is also known that, for every Banach space $X, C(K, X)$ (respectively, $L_{1}(\mu, X)$ ) has the Daugavet property if and only if $X$ has or $K$ is perfect (respectively, $\mu$ is atomless) (see [9]).

In this paper, we show that $L_{\infty}(\mu, X)$ has the Daugavet property if and only if $X$ has or the $\sigma$-finite measure $\mu$ is atomless. This extends an analogous result for $l_{\infty}(X)$ given in $[\mathbf{1 3}]$.

Throughout the paper, $(\Omega, \Sigma, \mu)$ stands for a $\sigma$-finite measure space and $X$ stands for an arbitrary Banach space. We write $L_{\infty}(\mu, X)$ for the Banach space of all equivalence classes of essentially bounded (Bochner) measurable functions from $\Omega$ into $X$, endowed with its natural norm

$$
\|f\|=\inf \{\lambda \geqslant 0:\|f(t)\| \leqslant \lambda \text { a.e. }\}
$$

for each $f \in L_{\infty}(\mu, X)$. To shorten the notation, we use the same letter to denote both a measurable function and its equivalence class. We refer to [5] for background on this topic.

\section{The results}

To generalize the fact given in $[\mathbf{9}]$ that $n\left(l_{\infty}(X)\right)=n(X)$, we require two preliminary results. The first one is well known for scalar-valued functions.

Lemma 2.1. Let $f \in L_{\infty}(\mu, X)$ with $\|f(t)\|>\lambda$ a.e. Then there exists $B \in \Sigma$ with $0<\mu(B)<\infty$ such that

$$
\left\|\frac{1}{\mu(B)} \int_{B} f(t) \mathrm{d} \mu(t)\right\|>\lambda .
$$

Proof. Since $f(\Omega)$ is essentially separable, we can certainly assume that $X$ is separable. Hence we can write

$$
X \backslash \lambda B_{X}=\bigcup_{n \in \mathbb{N}} B_{n},
$$


where $B_{n}$ are closed balls. Therefore, there exists $n \in \mathbb{N}$ such that $A=f^{-1}\left(B_{n}\right)$ has positive measure. Let $B \in \Sigma$ such that $B \subseteq A$ and $0<\mu(B)<\infty$. By convexity (see [5, Corollary II.2.8]),

$$
\frac{1}{\mu(B)} \int_{B} f(t) \mathrm{d} \mu(t)
$$

is contained in $B_{n}$, and the result follows.

Again according to the fact that every function in $L_{\infty}(\mu, X)$ is essentially separably valued, the following result, which we shall use throughout the proof of Theorem 2.3, follows immediately.

Lemma 2.2. Let $f \in L_{\infty}(\mu, X), C \in \Sigma$ with positive measure, and $\varepsilon>0$. Then there exist $x \in X$ and $A \subseteq C$ with $0<\mu(A)<\infty$ such that $\|x\|=\left\|f \chi_{C}\right\|$ and $\left\|(f-x) \chi_{A}\right\|<\varepsilon$. Accordingly, the set

$$
\left\{x \chi_{A}+f \chi_{\Omega \backslash A}: x \in S_{X}, f \in B_{L_{\infty}(\mu, X)}, A \in \Sigma \text { with } 0<\mu(A)<\infty\right\}
$$

is dense in $S_{L_{\infty}(\mu, X)}$.

We can now state our main result.

Theorem 2.3. Let $(\Omega, \Sigma, \mu)$ be a $\sigma$-finite measure space and let $X$ be a Banach space. Then

$$
n\left(L_{\infty}(\mu, X)\right)=n(X) .
$$

Proof. In order to show that $n\left(L_{\infty}(\mu, X)\right) \geqslant n(X)$, we fix $T \in L\left(L_{\infty}(\mu, X)\right)$ with $\|T\|=1$. The procedure is to prove that $v(T) \geqslant n(X)$. Given $\varepsilon>0$, we may find $f \in S_{L_{\infty}(\mu, X)}, x_{0} \in S_{X}$, and $A, B \in \Sigma$ with $0<\mu(B)<\infty$, such that

$$
B \subseteq A \text { and }\left\|\frac{1}{\mu(B)} \int_{B} T\left(x_{0} \chi_{A}+f \chi_{\Omega \backslash A}\right) \mathrm{d} \mu\right\|>1-\varepsilon .
$$

Indeed, take $f \in S_{L_{\infty}(\mu, X)}$ and $C \subseteq \Omega$ with $\mu(C)>0$ such that

$$
\|[T f](t)\|>1-\frac{1}{2} \varepsilon \quad(t \in C) .
$$

On account of Lemma 2.2, there exist $y_{0} \in B_{X}$ and $A \subseteq C$ with $\mu(A)>0$ such that $\left\|\left(f-y_{0}\right) \chi_{A}\right\|<\frac{1}{2} \varepsilon$. Now, write $y_{0}=\lambda x_{1}+(1-\lambda) x_{2}$ with $0 \leqslant \lambda \leqslant 1, x_{1}, x_{2} \in S_{X}$, and consider the functions

$$
f_{j}=x_{j} \chi_{A}+f \chi_{\Omega \backslash A} \in L_{\infty}(\mu, X) \quad(j=1,2),
$$

which clearly satisfy $\left\|f-\left(\lambda f_{1}+(1-\lambda) f_{2}\right)\right\|<\frac{1}{2} \varepsilon$. Since $A \subseteq C$, by using (2.2), we have

$$
\left\|\left[T f_{1}\right](t)\right\|>1-\varepsilon \quad \text { or } \quad\left\|\left[T f_{2}\right](t)\right\|>1-\varepsilon
$$

for every $t \in A$. Now, we choose $i \in\{1,2\}$ such that

$$
A_{i}=\left\{t \in A:\left\|\left[T f_{i}\right](t)\right\|>1-\varepsilon\right\}
$$


has positive measure, we write $x_{0}=x_{i}$, and finally we use Lemma 2.1 to get $B \subseteq A_{i} \subseteq A$, satisfying our requirements.

Next we fix $x_{0}^{*} \in S_{X^{*}}$ with $x_{0}^{*}\left(x_{0}\right)=1$, we write

$$
\Phi(x)=x \chi_{A}+x_{0}^{*}(x) f \chi_{\Omega \backslash A} \in L_{\infty}(\mu, X) \quad(x \in X),
$$

and we consider the operator $S \in L(X)$ given by

$$
S x=\frac{1}{\mu(B)} \int_{B} T(\Phi(x)) \mathrm{d} \mu \quad(x \in X) .
$$

According to (2.1), we have $\|S\| \geqslant\left\|S x_{0}\right\|>1-\varepsilon$. So we may find $x \in S_{X}$ and $x^{*} \in S_{X^{*}}$ such that

$$
x^{*}(x)=1 \quad \text { and } \quad\left|x^{*}(S x)\right| \geqslant n(X)[1-\varepsilon] .
$$

Set $g=\Phi(x) \in S_{L_{\infty}(\mu, X)}$ and define the functional $g^{*} \in S_{L_{\infty}(\mu, X)^{*}}$ by

$$
g^{*}(h)=x^{*}\left(\frac{1}{\mu(B)} \int_{B} h \mathrm{~d} \mu\right) \quad\left(h \in L_{\infty}(\mu, X)\right) .
$$

Since $B \subseteq A$, we have $g^{*}(g)=1$ and

$$
\left|g^{*}(T g)\right|=\left|x^{*}(S x)\right| \geqslant n(X)[1-\varepsilon] .
$$

Hence $v(T) \geqslant n(X)$, as required.

For the reverse inequality, we fix $S \in L(X)$ with $\|S\|=1$ and define $T \in L\left(L_{\infty}(\mu, X)\right)$ by

$$
[T(f)](t)=S(f(t)) \quad\left(t \in \Omega, f \in L_{\infty}(\mu, X)\right) .
$$

Then $\|T\|=1$ and so $v(T) \geqslant n\left(L_{\infty}(\mu, X)\right)$. According to Lemma 2.2 together with [2, Theorem 9.3], given $\varepsilon>0$ there exist $x \in S_{X}, f \in B_{L_{\infty}(\mu, X)}, A \in \Sigma$ with $0<\mu(A)<\infty$, and $x^{*} \in S_{X^{*}}$ with $x^{*}(x)=1$ such that

$$
v(T)-\varepsilon<\left|x^{*}\left(\frac{1}{\mu(A)} \int_{A} T\left(x \chi_{A}+f \chi_{\Omega \backslash A}\right) \mathrm{d} \mu\right)\right| .
$$

On the other hand,

$$
\frac{1}{\mu(A)} \int_{A} T\left(x \chi_{A}+f \chi_{\Omega \backslash A}\right) \mathrm{d} \mu=S\left(\frac{1}{\mu(A)} \int_{A}\left(x \chi_{A}+f \chi_{\Omega \backslash A}\right) \mathrm{d} \mu\right)=S x .
$$

Therefore,

$$
n\left(L_{\infty}(\mu, X)\right)-\varepsilon \leqslant v(T)-\varepsilon<\left|x^{*}(S x)\right| \leqslant v(S)
$$

and so $n(X) \geqslant n\left(L_{\infty}(\mu, X)\right)$.

The last part of the paper is dedicated to the study of the Daugavet property for $L_{\infty}(\mu, X)$. To this end, we need a characterization of this property given in [12, Corollary 2.3]. 
Lemma 2.4. $X$ has the Daugavet property if and only if for every $x \in S_{X}$ and every $\varepsilon>0$

$$
B_{X}=\overline{\operatorname{co}}\left\{y \in B_{X}:\|x-y\| \geqslant 2-\varepsilon\right\} .
$$

Since the proof of the non-easy part of the following result is analogous to that given in [12] for $C(K, X)$, it should be known to experts. However, we could not find it in the journal literature.

Theorem 2.5. Let $(\Omega, \Sigma, \mu)$ be a $\sigma$-finite measure space and let $X$ be a Banach space. Then $L_{\infty}(\mu, X)$ has the Daugavet property if and only if $X$ has or $\mu$ is atomless.

Proof. Let us first suppose that $\mu$ is atomless. Set $f \in S_{L_{\infty}(\mu, X)}, \varepsilon>0$ and $B \in \Sigma$ with

$$
\mu(B)>0 \quad \text { and } \quad\|f(t)\|>1-\frac{1}{2} \varepsilon \quad(t \in B) .
$$

Given $h \in S_{L_{\infty}(\mu, X)}$ and $n \in \mathbb{N}$, we take $B_{1}, \ldots, B_{n}$ pairwise disjoint subsets of $B$ with positive measure and we consider the function

$$
g_{j}=h \chi_{\Omega \backslash B_{j}}-f \chi_{B_{j}} \in B_{L_{\infty}(\mu, X)}
$$

for each $j \in\{1, \ldots, n\}$. For every $t \in B_{j}$ we have

$$
\left\|h(t)-\frac{1}{n} \sum_{i=1}^{n} g_{i}(t)\right\|=\frac{1}{n}\|h(t)+f(t)\| \leqslant \frac{2}{n},
$$

and for $t \notin \bigcup_{j=1}^{n} B_{j}$ we have

$$
h(t)=\frac{1}{n} \sum_{i=1}^{n} g_{i}(t)
$$

Since $\left\|f-g_{j}\right\|>2-\varepsilon$, the above lemma shows that $L_{\infty}(\mu, X)$ has the Daugavet property.

To finish the proof, we write $L_{\infty}(\mu, X)$ in the form

$$
L_{\infty}(\nu, X) \oplus_{\infty}\left[\bigoplus_{i \in I} X\right]_{l_{\infty}}
$$

for a suitable set $I \subseteq \mathbb{N}$ and an atomless measure $\nu$. Now, it should be noted that an $l_{\infty}$-sum of Banach spaces has the Daugavet property if and only if every summand has $[\mathbf{1 3}]$.

Acknowledgements. Research partly supported by Spanish MCYT projects BFM2000-1467 and BFM2002-00061.

\section{References}

1. Y. A. Abramovich, C. D. Aliprantis and O. Burkinshaw, The Daugavet equation in uniformly convex Banach spaces, J. Funct. Analysis 97 (1991), 215-230.

2. F. F. Bonsall AND J. DunCAN, Numerical ranges of operators on normed spaces and of elements of normed algebras, London Mathematical Society Lecture Notes Series, vol. 2 (London Mathematical Society, Cambridge, 1971). 
3. F. F. Bonsall And J. Duncan, Numerical ranges II, London Mathematical Society Lecture Notes Series, vol. 10 (London Mathematical Society, Cambridge, 1973).

4. I. K. DAugavet, On a property of completely continuous operators in the space $C, U s p$. Mat. Nauk 18 (1963), 157-158 (in Russian).

5. J. Diestel And J. J. Uhl, Vector measures, Mathematical Surveys, vol. 15 (American Mathematical Society, Providence, RI, 1977).

6. V. M. Kadets, R. V. Shvidkoy, G. G. Sirotkin and D. Werner, Banach spaces with the Daugavet property, Trans. Am. Math. Soc. 352 (2000), 855-873.

7. G. López, M. Martín And R. PAyÁ, Real Banach spaces with numerical index 1, Bull. Lond. Math. Soc. 31 (1999), 207-212.

8. M. MARTín, A survey on the numerical index of a Banach space, Extracta Math. 15 (2000), 265-276.

9. M. MARtín AND R. PAYÁ, Numerical index of vector-valued function spaces, Studia Math. 142 (2000), 269-280.

10. T. OIKHBERG, The Daugavet property of $C^{*}$-algebras and non-commutative $L_{p}$-spaces, Positivity 6 (2002), 59-73.

11. R. V. Shvidkoy, Geometric aspects of the Daugavet property, J. Funct. Analysis 176 (2000), 198-212.

12. D. Werner, Recent progress on the Daugavet property, Irish Math. Soc. Bull. 46 (2001), 77-97.

13. P. Wojtaszczyk, Some remarks on the Daugavet equation, Proc. Am. Math. Soc. 115 (1992), 1047-1052. 